\title{
Targeting the COX2/MET/TOPK signaling axis induces apoptosis in gefitinib-resistant NSCLC cells
}

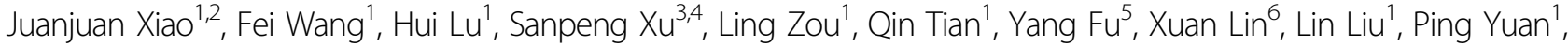 \\ Xiaofang $\mathrm{Ni}^{1}$, Tengfei $\mathrm{Ma}^{1}$, Fanfan Zeng ${ }^{1}$, Peipei Xue ${ }^{7}$, Ruijuan $\mathrm{Xiu}^{1}$, Jianmin Zhang ${ }^{1}$, Xinying $\mathrm{Ji}^{8}$, Hongbo Hu${ }^{9}$, \\ Shangyun Lu' ${ }^{9}$, Hongtian Dai ${ }^{10}$, Yuan $\mathrm{Li}^{11}$, Qian $\mathrm{Chu}^{12}$, Xia Zhao ${ }^{3,4}$, Qiuhong Duan ${ }^{1}$ and Feng Zhu ${ }^{1,2}$
}

\begin{abstract}
MET overactivation is one of the crucial reasons for tyrosine kinase inhibitor (TKI) resistance, but the mechanisms are not wholly clear. Here, COX2, TOPK, and MET expression were examined in EGFR-activating mutated NSCLC by immunohistochemical $(\mathrm{IHC}$ ) analysis. The relationship between COX2, TOPK, and MET was explored in vitro and ex vivo. In addition, the inhibition of HCC827GR cell growth by combining COX2 inhibitor (celecoxib), TOPK inhibitor (pantoprazole), and gefitinib was verified ex vivo and in vivo. We found that COX2 and TOPK were highly expressed in EGFR-activating mutated NSCLC and the progression-free survival (PFS) of triple-positive (COX2, MET, and TOPK) patients was shorter than that of triple-negative patients. Then, we observed that the COX2-TXA 2 signaling pathway modulated MET through AP-1, resulting in an inhibition of apoptosis in gefitinib-resistant cells. Moreover, we demonstrated that MET could phosphorylate TOPK at Tyr74 and then prevent apoptosis in gefitinib-resistant cells. In line with these findings, the combination of celecoxib, pantoprazole, and gefitinib could induce apoptosis in gefitinibresistant cells and inhibit tumor growth ex vivo and in vivo. Our work reveals a novel COX2/MET/TOPK signaling axis that can prevent apoptosis in gefitinib-resistant cells and suggests that a triple combination of FDA-approved drugs would provide a low-cost and practical strategy to overcome gefitinib resistance.
\end{abstract}

\section{Introduction}

Lung cancer is the most frequent cancer and the most common cause of cancer-related deaths worldwide ${ }^{1}$. Nonsmall cell lung cancer (NSCLC) represents $~ 80 \% \sim 90 \%$ of all lung cancers. Approximately $10-50 \%$ of patients with NSCLC harbor EGFR-activating mutations such as inframe deletions in exon 19 (Ex19del, particularly E746A750del) or missense mutation in exon 21 (L858R $)^{2}$. Although NSCLC with EGFR-activating mutations

Correspondence: Qian Chu (qianchu@tjh.tjmu.edu.cn) or Xia Zhao (yespeony@126.com) or Qiuhong Duan (duanqhwz@hust.edu.cn) or Feng Zhu (fengzhu@hust.edu.cn)

${ }^{1}$ Department of Biochemistry and Molecular Biology, School of Basic Medicine, Huazhong University of Science and Technology, Wuhan, Hubei 430030, China ${ }^{2}$ Cancer Research Institute, The Affiliated Hospital of Guilin Medical University, Guilin, Guangxi 541000, China

Full list of author information is available at the end of the article. These authors contributed equally: Juanjuan Xiao and Fei Wang Edited by M. Agostini exhibits sensitivity to epidermal growth factor receptor (EGFR) tyrosine kinase inhibitors (EGFR-TKIs), such as gefitinib and erlotinib, most patients treated with EGFRTKIs develop resistance within 10-14 months ${ }^{3}$.

MET (hepatocyte growth factor receptor, HGFR) overactivation is one of the crucial reasons for TKI resistance. It has been reported that $M E T$ gene amplification, activating gene mutations, and MET overexpression can lead to MET overactivation, and that these changes were all significantly associated with unfavorable prognosis in NSCLC $^{4,5}$. MET encodes a transmembrane tyrosine kinase receptor for hepatocyte growth factor (HGF) and it can also be activated by HGF or by the Src/FAK or EGFR signaling pathway in lung cancer ${ }^{6,7}$. Activated MET interacts with several adaptor proteins, such as STAT3, PI3K, or Src, subsequently activating the mitogenactivated protein kinase and mammalian target of rapamycin pathways to mediate proliferation, apoptosis, and

\section{(c) The Author(s) 2019}

(c) (i) Open Access This article is licensed under a Creative Commons Attribution 4.0 International License, which permits use, sharing, adaptation, distribution and reproduction cc) in any medium or format, as long as you give appropriate credit to the original author(s) and the source, provide a link to the Creative Commons license, and indicate if changes were made. The images or other third party material in this article are included in the article's Creative Commons license, unless indicated otherwise in a credit line to the material. If material is not included in the article's Creative Commons license and your intended use is not permitted by statutory regulation or exceeds the permitted use, you will need to obtain permission directly from the copyright holder. To view a copy of this license, visit http://creativecommons.org/licenses/by/4.0/. 
migration $^{8}$. However, the existing molecular regulatory mechanisms and downstream events are not sufficient to explain the pathogenic factors of MET-mediated drug resistance and relapse. More information is needed to explore MET involvement in pathogenesis.

COX2 is overexpressed in inflammatory tissue and many neoplastic tissues ${ }^{9}$. Some studies indicate that its overexpression could result in increased prostaglandin (PG) production and secretion, which in turn activates growth pathways and inhibits apoptotic pathways ${ }^{10}$. COX2 can modulate EGFR transcription through nuclear factor activator protein-1 (AP-1) and promote the malignant transformation of preneoplastic cells ${ }^{11}$. In addition, it was reported that AP-1 could mediate the transcriptional activation of $\mathrm{MET}^{12}$. Therefore, whether COX2 could regulate MET transcription through AP-1inducing TKI resistance drew our attention.

T-lymphokine-activated killer cell-originated protein kinase (TOPK) is associated with histological type, lymph node metastasis, and TNM stage, and is positively correlated with Ki67 and p53 expression in $\mathrm{NSCLC}^{13}$. Recently, Li et al. ${ }^{14}$ reported that TOPK silencing inhibited the growth and enhanced the gefitinib sensitivity of lung cancer cells, and TOPK is considered a potential therapeutic target and a prognostic marker for lung cancer. Our previous research found that phosphorylation of TOPK at Y74 and Y272 sites by Src promoted the tumorigenesis of colon cancer ${ }^{15}$. MET is a tyrosine kinase and whether it is upstream of TOPK is unknown. Therefore, the relationship between MET and TOPK in TKI resistance should be studied further.

Here we found that the COX2-TXA 2 signaling pathway promoted MET transcription through AP-1 and MET phosphorylated TOPK at the Y74 site. Moreover, the activation of this pathway prevented apoptosis and promoted gefitinib resistance in NSCLC. On this basis, the triple combination of COX2 inhibitor celecoxib ${ }^{16}$, TOPK inhibitor pantoprazole ${ }^{17}$, and gefitinib was proven to induce apoptosis in gefitinib-resistant cells and inhibit tumor growth in vitro and in vivo. Our findings may provide a new practical strategy for NSCLC patients with TKI resistance.

\section{Materials and methods}

\section{Antibodies and reagents}

TOPK (sc-293028) and p-AKT (sc-7985) were purchased from Santa Cruz Technology, Inc. (Santa Cruz, CA). $\beta$-Actin (ET1701-80) was purchased from Hangzhou HuaAn Biotechnology, Inc. (Hangzhou, ZJ). Antibodies to detect COX2 (\#4842), MET (\#8198), p-MET (\#3077), pERK (\#9101), ERK (\#9102), AKT (\#9272), p-c-Jun (\#2361), c-Jun (\#9165), PARP (\#9542), c-PARP (\#5625), His (\#2365), HA (\#3724), and p-Tyrosine (\#8954) were purchased from Cell Signaling Technology (Danvers, MA). Horseradish peroxidase-labeled goat anti-mouse IgG $(\mathrm{H}+\mathrm{L})(\mathrm{E} 031110)$ and goat anti-rabbit IgG $(\mathrm{H}+\mathrm{L})(\mathrm{E} 031120)$ were purchased from EarthOx, LLC (San Francisco, CA). PhosphoTOPK at Y74 antibody was prepared by Abgent, Inc. (Suzhou, JS). All antibodies were used following the instructions of the respective manufacturers.

Gentamicin, L-glutamine, epidermal growth factor, HGF, Tris, $\mathrm{NaCl}$, and SDS for molecular biology were purchased from Sigma-Aldrich (St. Louis, MO). U46619, Seratrodast, and Ozagrel $\mathrm{HCl}$ were all obtained from ApexBio Technology, LLC (Houston, TX). Celecoxib was purchased from Pfizer (New York, NY). Gefitinib was purchased from Gold Biotechnology (St. Louis, MO). Pantoprazole was obtained from Kaifu Co., Ltd (Wuxi, JS). SR11302 and PGE2 were purchased from Selleckchem, Inc. (Houston, TX).

\section{Cell culture and transfection}

HCC827, MRC-5, H1299, A549, H1650, H1975, and HEK293T cells were purchased from American Type Culture Collection (ATCC; Manassas, VA). The gefitinibresistant HCC827 (HCC827GR) cell line was kindly provided by Dr. Pasi A. Jänne (Harvard Medical School, Boston, MA) and was authenticated by the Jänne group ${ }^{18}$. Cell lines were authenticated by periodic short tandem repeat profiling and mycoplasma-negative status was confirmed before freezing. All cell lines were thawed and passaged at least three times before initiating experiments. Cells were discarded after 18 passages. Cells were cultured at $37^{\circ} \mathrm{C}$ in a $5 \% \mathrm{CO}_{2}$ humidified incubator following the ATCC protocols. MRC-5 human normal lung fibroblasts were grown in Eagle's minimum essential medium (Gibco, Grand Island, NY) with $10 \%$ fetal bovine serum (FBS; Gibco, Grand Island, NY) and A549 human lung cancer cells were cultured with F-12K medium (Sigma, St. Louis, MO) containing 10\% FBS. All other human lung cancer cells were grown in RPMI-1640 medium (Gibco, Grand Island, NY) supplemented with 10\% FBS. HEK293T cells (stably expressing the SV40 large T antigen in HEK293 cells) were cultured in Dulbecco's modified Eagles' medium (Gibco, Grand Island, NY) supplemented with 10\% FBS.

When cells reached $60 \%$ confluence, transfection was performed using Simple-fect (Signaling Dawn Biotech, Wuhan, HB) following the manufacturer's instructions. For stable transfection experiments, G418 (Sigma, St. Louis, MO) or hygromycin (Thermo Fisher, Waltham, MA) was added for stable clone selection. After 3 weeks, the individual clones were ring-isolated. Expression of the protein was verified by western blotting analysis.

\section{Clinical data and IHC analysis of a tissue array}

One hundred fifty-three cases of NSCLC with activating mutations in the EGFR gene, including a deletion in exon 19 and an L858R mutation in exon 21, were collected 
from archival files of Wuhan Tongji Hospital dating from 2013 to 2016. NSCLC specimens and matched adjacent normal tissues were used to construct a tissue microarray (TMA). Ethical approval was obtained from the medical ethics committee of Tongji Medical College, Huazhong University of Science and Technology. Paraffin-embedded tissues were sectioned at $5 \mu \mathrm{m}$ thickness. Slides were baked at $60^{\circ} \mathrm{C}$ for $1 \mathrm{~h}$ and were deparaffinized, rehydrated, and treated with $3 \%$ hydrogen peroxide for $10 \mathrm{~min}$. Antigen retrieval was performed in citrate buffer $\mathrm{pH} 6.0$ in a steamer for $2 \mathrm{~min}$ or in Tris-EDTA buffer $\mathrm{pH} 9.0$ at $100{ }^{\circ} \mathrm{C}$ for $20 \mathrm{~min}$. After the slides were blocked with $5 \%$ bovine serum albumin in phosphate-buffered saline (PBS) for $30 \mathrm{~min}$, tissue sections were incubated overnight at $4{ }^{\circ} \mathrm{C}$ with the indicated primary antibodies. The PBS and mouse or rabbit IgG1 (Santa Cruz Biotechnology, CA) were used as blank and negative controls. The sections were photographed using an Olympus Imaging System Microscope (BX51, Olympus, Tokyo). Immunohistochemical (IHC) staining was evaluated simultaneously by two pathologists who did not know the clinicopathological features of the patients.

\section{Western blotting analysis and immunoprecipitation}

Cells were collected and lysed in RIPA buffer $(1 \times$ PBS, $1 \%$ Nonidet P-40, $0.5 \%$ sodium deoxycholate, $0.1 \%$ SDS, $1 \mathrm{mmol} / \mathrm{L} \mathrm{Na}_{3} \mathrm{VO}_{4}$, and $1 \mathrm{mmol} / \mathrm{L}$ aprotinin and $1 \mathrm{mmol} / \mathrm{L}$ phenylmethylsulfonyl fluoride). Then, the samples were sonicated $15 \mathrm{~s}$ three times and centrifuged at 12,000 r.p.m. for $10 \mathrm{~min}$. The quantity of protein was determined by the Bradford method. After that, the samples were separated on a 7\%-15\% SDS-polyacrylamide gel electrophoresis (PAGE) gel, transferred onto a polyvinylidene difluoride membrane (Millipore, Billerica, MA) and subsequently visualized by chemiluminescence (BIO-RAD, Hercules, CA) in triplicate. The samples for immunoprecipitation were collected in $1 \%$ CHAPS (3-[(3-Cholamidopropyl)dimethylammonio]propanesulfonate) instead of RIPA buffer. Equal amounts of protein $(1-2 \mathrm{mg})$ were subjected to immunoprecipitation following the manufacturer's suggested protocol.

\section{Anchorage-independent cell transformation assay}

Different cell lines $\left(8 \times 10^{3} /\right.$ well $)$ in a six-well plate were exposed or not exposed to different drugs and cultured in $1 \mathrm{ml}$ of $0.33 \%$ BME (Eagle basal medium, Sigma, St. Louis, MO) agar (Sigma, St. Louis, MO) containing 10\% FBS over $3 \mathrm{ml}$ of $0.5 \%$ BME agar containing 10\% FBS. The cells were maintained in a $37^{\circ} \mathrm{C}, 5 \% \mathrm{CO}_{2}$ incubator for 5-10 days and then their colonies were counted and scored using Image-Pro Plus software.

\section{In vitro growth inhibition assay}

HCC827GR cells were seeded in each well of a 96-well plate and were cultured in RPMI-1640 medium with 10\%
FBS for $24 \mathrm{~h}$. Then, different drugs at the indicated doses were added to the medium for an additional 24,48 , or $72 \mathrm{~h}$. The inhibitory effects of different groups on cell growth were examined using the MTT (3-(4,5-dimethylthiazol-2-yl)-2,5-diphenyltetrazolium bromide) (Sigma, St. Louis, $\mathrm{MO}$ ) viability assay, according to the manufacturer's instructions. All the experiments were performed in triplicate and the mean absorbance values were calculated.

\section{Prostaglandin determination}

The measurement of PGs $\left(\mathrm{PGD}_{2}, \mathrm{PGE}_{2}, \mathrm{PGF}_{2}, \mathrm{PGI}_{2}\right.$, and $\mathrm{TXA}_{2}$ ) in the cell culture medium was conducted using enzyme immunoassay kits from Cayman Chemical Company (Ann Arbor, MI). In brief, cells were plated in six-well plates. When cells reached $80 \%$ confluence, $1 \mathrm{ml}$ fresh medium with or without reagents was added and cells were further incubated for a different time. Then, the supernatants were collected for PG measurement following the manufacturer's instructions.

\section{Flow cytometry analysis}

Cell apoptosis was determined using the Annexin $\mathrm{V}$-fluorescein isothiocyanate (FITC)/Propidium Iodide (PI) Apoptosis Detection Kit (Elabscience, Wuhan, HB) following the manufacturer's suggested protocol. Cells $\left(2 \times 10^{5} /\right.$ well $)$ were seeded in a six-well plate and cultured at $37^{\circ} \mathrm{C}$ and $5 \% \mathrm{CO}_{2}$ for $12 \mathrm{~h}$. After treatment with gefitinib for $24 \mathrm{~h}$, cells were collected and washed with PBS. Then, the cells were incubated for $15 \mathrm{~min}$ at room temperature with Annexin V-FITC plus PI according to the protocol. Apoptosis was analyzed by a FACSCalibur flow cytometer (BD Biosciences, San Jose, CA, USA).

\section{In vitro kinase assay}

The MET active kinase, ATP, and $10 \times$ kinase buffer were purchased from Millipore Corporation (Billerica, MA). HisTOPK-WT and His-TOPK-Y74F were expressed in Escherichia coli BL21 bacteria as before ${ }^{15}$. Peptides were synthesized by GL Biochem Ltd (Shanghai, SH). The inactive substrate $(2 \mu \mathrm{g})$ and the active kinase $(0.2 \mu \mathrm{g}$ in a $30 \mu \mathrm{l}$ reaction) were incubated at $32{ }^{\circ} \mathrm{C}$ for $1 \mathrm{~h}$ in $1 \times$ kinase buffer containing $100 \mu \mathrm{mol} / \mathrm{L}$ ATP or $1 \mu \mathrm{Ci}\left[\gamma^{-32}{ }^{32} \mathrm{P}\right.$-ATP (China Isotope \& Radiation Corporation, Beijing, BJ). The samples were added to $5 \times$ SDS buffer, and then resolved by SDSPAGE and visualized by autoradiography or western blot.

\section{Combined drug analysis}

The combined effect was evaluated by MTT assay at the nonconstant ratio of each drug. CompuSyn (ComboSyn, Inc., Paramus, NJ) was used to assess the interaction of the drug combinations for synergy/additivity/antagonism using the Combination index $(\mathrm{CI})$ value from the Chou-Talalay $\operatorname{method}^{19}$. CI values of $<1,1$, and $>1$ indicate synergistic, additive, and antagonistic effects, respectively. 


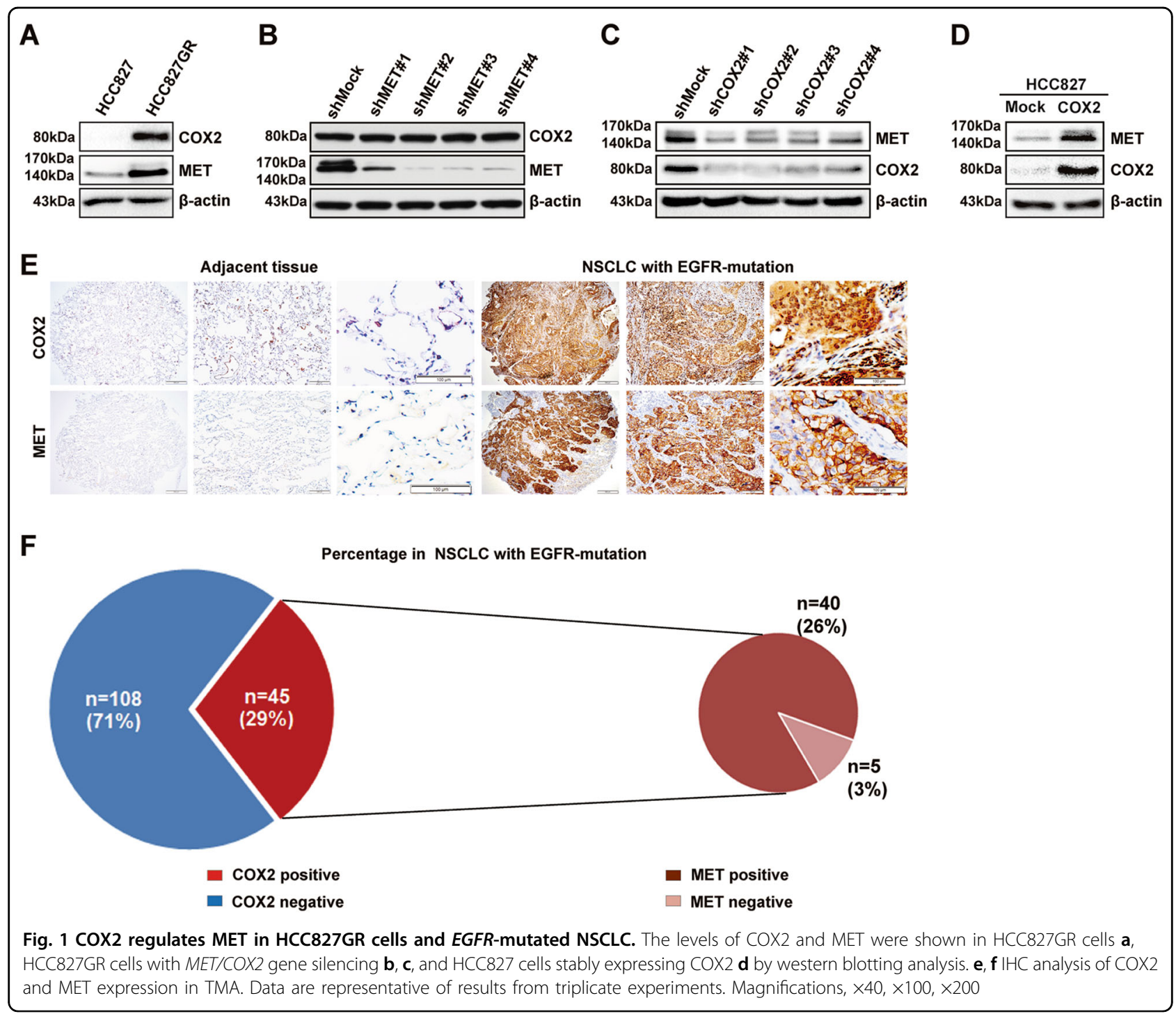

\section{In vivo study}

Male NOD-Prkdc ${ }^{\text {scid }}$ Il2rg ${ }^{\text {null }}$ (NPSG) mice $(18-20$ g, 6 weeks of age, Weishanglituo Company, Beijing) were randomly divided into three groups ( $n=10$ per group). HCC827GR cells $\left(5 \times 10^{6} / 0.1 \mathrm{ml}\right.$ RPMI-1640 medium, $0.1 \mathrm{ml}$ Matrigel) were inoculated subcutaneously into the right flank of each mouse. When tumors reached an average volume of $100 \mathrm{~mm}^{3}$, vehicle control, gefitinib $(20 \mathrm{mg} / \mathrm{kg})$, or gefitinib $(20 \mathrm{mg} / \mathrm{kg})$ plus celecoxib $(30 \mathrm{mg} /$ $\mathrm{kg})$ plus pantoprazole $(150 \mathrm{mg} / \mathrm{kg})$ was administered by intraperitoneal injection three times a week for 3 weeks. Double blinding was done in this experiment. Tumor volume was calculated using the ellipsoid formula (length $\times$ width $\times$ height $\times 0.52$ ). Animal maintenance and experimental procedures were approved by the Animal Care Committee of Wuhan Servicebio Technology Co., Ltd (Wuhan, China).

\section{Statistical analysis}

All quantitative data except for the combined drug analysis are expressed as the mean values \pm SD of at least three independent experiments or samples. Significant differences were determined by Student's $t$-test or one-way analysis of variance. The Pearson's correlation was used to measure the strength of association between two variables. All statistical tests were two-sided and $P<0.05$ was considered significant $\left({ }^{*} P<0.05,{ }^{* *} P<0.01,{ }^{* * * *} P<0.001\right)$.

\section{Results}

COX2 is the upstream regulator of MET in HCC827GR cells and EGFR-activating mutated NSCLC

To confirm whether COX2 could regulate MET expression in NSCLC, we first analyzed the expression of COX2 in HCC827 and HCC827GR cells (MET amplification, gefitinib resistance ${ }^{18}$ ). The results in Fig. 1a 


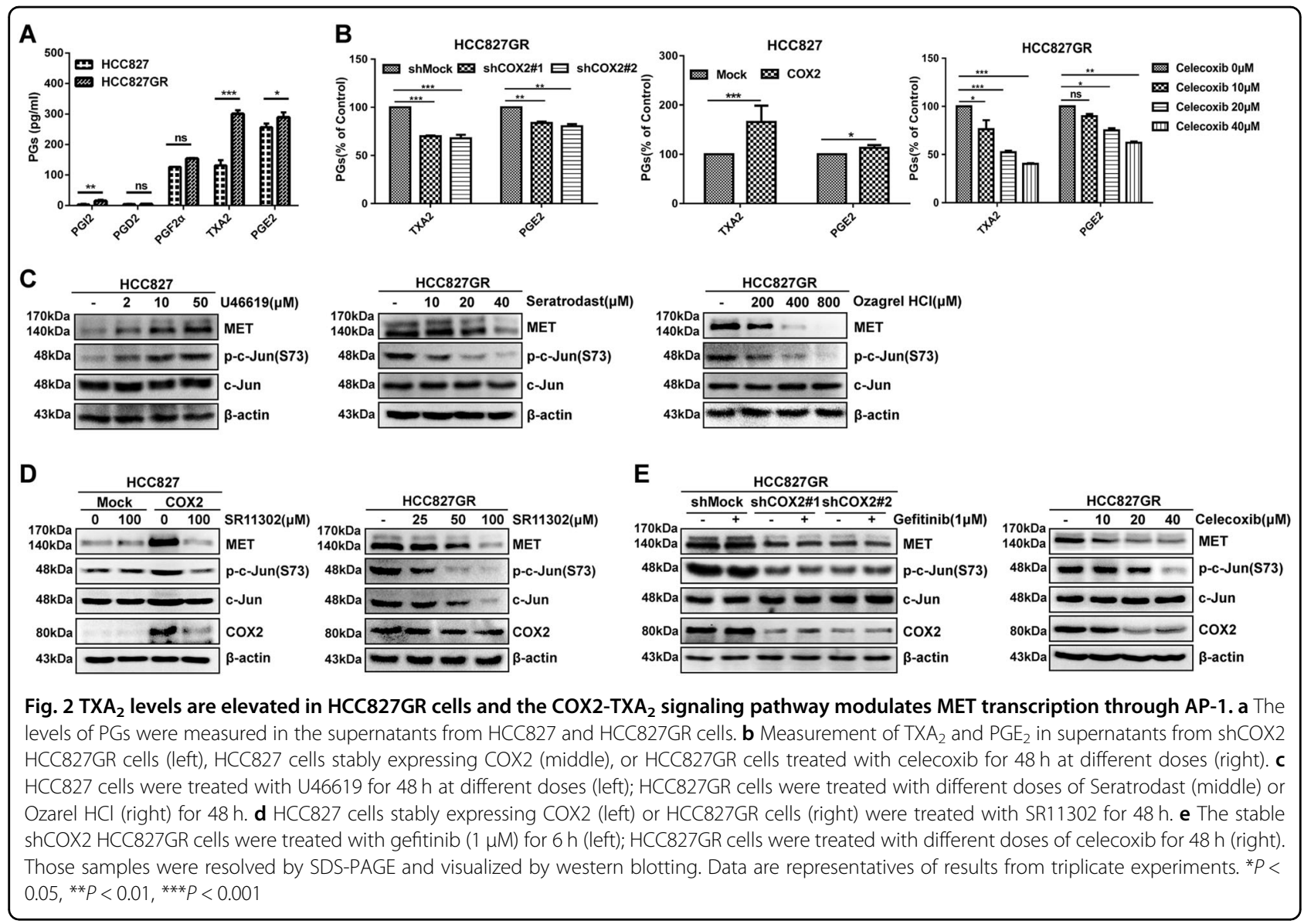

showed that COX2 expression was significantly higher in HCC827GR cells than in HCC827 cells. After MET was knocked down in HCC827GR cells, the expression of COX2 did not change (Fig. 1b). However, after silencing COX2 in HCC827GR cells, the expression of MET was dramatically decreased (Fig. 1c). In turn, the expression of MET was increased in HCC827 cells stably overexpressing COX2 (Fig. 1d). These results suggested that COX2 acted as an upstream regulator of MET in HCC827GR cells, but MET could not regulate COX2.

Next, we further examined the expression of COX2 and MET in the TMA as described previously by IHC staining. The results showed that the protein levels of COX2 or MET were increased in EGFR-activating mutated NSCLC compared with adjacent tissues (Fig. 1e). The positive expression rate of COX2 was $29 \%$ in these NSCLC patients and it was especially noteworthy that the positive expression rate of MET was as high as $89 \%$ in the COX2positive NSCLC patients (Fig. 1f). This finding revealed that COX2 could also be an upstream regulator of MET in NSCLC patients with both COX2 and MET-high expression.
$\mathrm{TXA}_{2}$ levels were significantly elevated in HCC827GR cells and the COX2-TXA ${ }_{2}$ signaling pathway modulates MET transcription through AP-1

COX2, as a rate-limiting enzyme, can convert arachidonic acid to $\mathrm{PGs}^{20}$. To further explore which PGs participated in the regulation of MET, five major bioactive PGs of the supernatant fractions of HCC 827 and HCC827GR cells were measured by enzyme-linked immunosorbent assay. The results showed that $\mathrm{TXA}_{2}$ and $\mathrm{PGE}_{2}$ were the abundant PGs in HCC827GR cells. Compared with those in HCC827 cells, the levels of $\mathrm{TXA}_{2}, \mathrm{PGE}_{2}$, and $\mathrm{PGI}_{2}$ were significantly elevated in HCC827GR cells, whereas $\mathrm{PGF}_{2 \alpha}$ and $\mathrm{PGD}_{2}$ levels did not change significantly. Intriguingly, the $\mathrm{TXA}_{2}$ level changed the most, with a striking threefold enhancement (Fig. 2a). Furthermore, the levels of $\mathrm{TXA}_{2}$ and $\mathrm{PGE}_{2}$ (Fig. 2b left) from the supernatant fractions of HCC827GR cells with shCOX2 were decreased and COX2 overexpression in HCC827 cells increased the levels of TXA 2 and $\mathrm{PGE}_{2}$ (Fig. $2 \mathrm{~b}$, middle). After HCC827GR cells were treated with celecoxib, the levels of $\mathrm{TXA}_{2}$ and $\mathrm{PGE}_{2}$ gradually decreased in a dose-dependent manner (Fig. 2b, right). 
Based on the above results, the high levels of TXA 2 and PGE2, especially $\mathrm{TXA}_{2}$, may play essential roles in HCC827GR cells. To confirm the role of TXA ${ }_{2}$ in gefitinib resistance, three experiments were designed. First, HCC827 cells were treated with different concentrations of $\mathrm{TXA}_{2}$ receptor agonist (U46619) for $48 \mathrm{~h}$ and the level of MET was detected by western blotting. The results showed that the expression of MET gradually increased in a dose-dependent manner after U46619 treatment (Fig. 2c, left). Second, the expression of MET was gradually decreased in a dose-dependent manner in HCC827GR cells treated with $\mathrm{TXA}_{2}$ receptor antagonist (Seratrodast) or the inhibitor of $\mathrm{TXA}_{2}$ synthase (Ozagrel $\mathrm{HCl}$ ) for $48 \mathrm{~h}$ (Fig. 2c, middle or right). These results suggest that among five PGs, $\mathrm{TXA}_{2}$ is the critical mediator that regulates MET expression in HCC827GR cells.

Previously, it was reported that AP-1 was one of the potential transcription factors for $\mathrm{MET}^{12}$. Moreover, the $\mathrm{TXA}_{2}$ receptor could lead to the activation of AP- 1 by activating a protein kinase $\mathrm{C}$-dependent pathway ${ }^{21}$. We hypothesized that the COX2- $\mathrm{TXA}_{2}$ signaling pathway might modulate MET transcription through AP-1. To verify whether the $\mathrm{COX} 2-\mathrm{TXA}_{2}$ signaling pathway increases MET expression through AP-1 in HCC827GR cells, we tested the AP-1 activity of HCC827GR cells using a phospho-c-jun (S73) antibody by western blotting. The results showed that the level of p-c-jun (S73) was enhanced in a dose-dependent way with U46619 treatment and attenuated with Seratrodast or Ozagrel $\mathrm{HCl}$ treatment (Fig. 2c). Meanwhile, the expression of MET was also strikingly decreased in HCC827GR cells or HCC827 cells stably overexpressing COX2 with AP-1 inhibitor (SR11302) treatment (Fig. 2d). Lower AP-1 activity and MET levels were observed in COX2 knockdown or celecoxib-treated HCC827GR cells (Fig. 2e). Hence, these results verified that the COX2-TXA 2 signaling pathway increased MET expression through AP-1.

\section{The expressions of TOPK and MET are positively correlated} in HCC827GR cells and EGFR-activating mutated NSCLC

TOPK was involved in the growth of lung cancer cells and could be activated by tyrosine kinase $\mathrm{Src}^{14,15}$. Therefore, the role of TOPK in gefitinib-resistant NSCLC with MET overactivation attracted our attention. Our results showed that TOPK and p-TOPK (Y74) levels were much higher in HCC827GR cells than in other lung cancer cell lines and a normal lung cell line (Fig. 3a). Unlike that in the parental HCC827 cells, the phosphorylation of TOPK, MET, and ERK1/2 in the HCC827GR cells was maintained in the presence of gefitinib (Fig. 3b). In addition, IHC analysis showed that the protein level of TOPK was increased in EGFRactivating mutated NSCLC compared with that in adjacent tissues (Fig. 3c). TOPK staining was positive in 121 (79\%) of 153 samples. Importantly, MET and TOPK were highly and positively correlated $(p<0.0001$; $R=0.1801)$ in EGFR-activating mutated NSCLC patients (Fig. 3d). These results demonstrated that the increased activity of TOPK is related to gefitinib resistance, and that the expression of TOPK has a positive correlation with that of MET in EGFR-activating mutated NSCLC.

\section{MET phosphorylates TOPK at the $\mathrm{Y} 74$ site in vitro and ex vivo}

Next, we wondered whether MET, as a tyrosine kinase, could phosphorylate TOPK directly. The results of the in vitro kinase assay indicated that MET could phosphorylate TOPK in vitro (Fig. 4a). The potential tyrosine phosphorylation sites of TOPK were predicted by NetPhos 3.0 (Fig. 4b). Five high-scoring peptides were synthesized commercially (Y74, Y131, Y271, Y272, and Y290) and individually incubated with active MET in the presence of $\left[\gamma^{-}{ }^{32} \mathrm{P}\right]$ ATP in an in vitro kinase assay. The data showed that the Y74 peptide of TOPK was notably phosphorylated by MET (Fig. 4c). Subsequently, the wildtype TOPK (WT) and Y74F TOPK (74F) proteins were purified from $E$. coli and were used as substrates for active MET in an in vitro kinase assay. Western blotting analysis using the prepared specific p-TOPK (Y74) antibody confirmed that MET could phosphorylate TOPK at the Y74 site (Fig. 4d).

We further verified that MET could phosphorylate TOPK in cells. First, MET was overexpressed in HEK293T cells and then the cells were stimulated with HGF. The results showed that the phosphorylation level of endogenous TOPK at Y74 was gradually increased in a dose-dependent manner with increasing amounts of transfected MET plasmid (Fig. 4e). Then, TOPK WT plasmid (HA-TOPK WT) or HA-TOPK 74F plasmid was cotransfected into HEK293T cells with MET plasmid (His-MET). The results showed that the level of $\mathrm{p}$-TOPK (Y74) increased in the group cotransfected with HATOPK WT (Fig. 4f). Subsequently, endogenous TOPK was immunoprecipitated from HCC827GR cells and then MET was detected by western blotting. The results indicated that TOPK could coimmunoprecipitate with MET in HCC827GR cells (Fig. 4g). After that, the level of endogenous p-TOPK (Y74) was decreased in MET knockdown HCC827GR cells (Fig. 4h). Moreover, it was also revealed that the level of p-TOPK (Y74) was increased in TOPK-WT-overexpressing HCC827 cells and the level of p-TOPK (Y74) was obviously decreased in TOPK-74F cells (Fig. 4i). These results thus far indicated that the phosphorylation of TOPK by MET at the Y74 site existed in cells. 


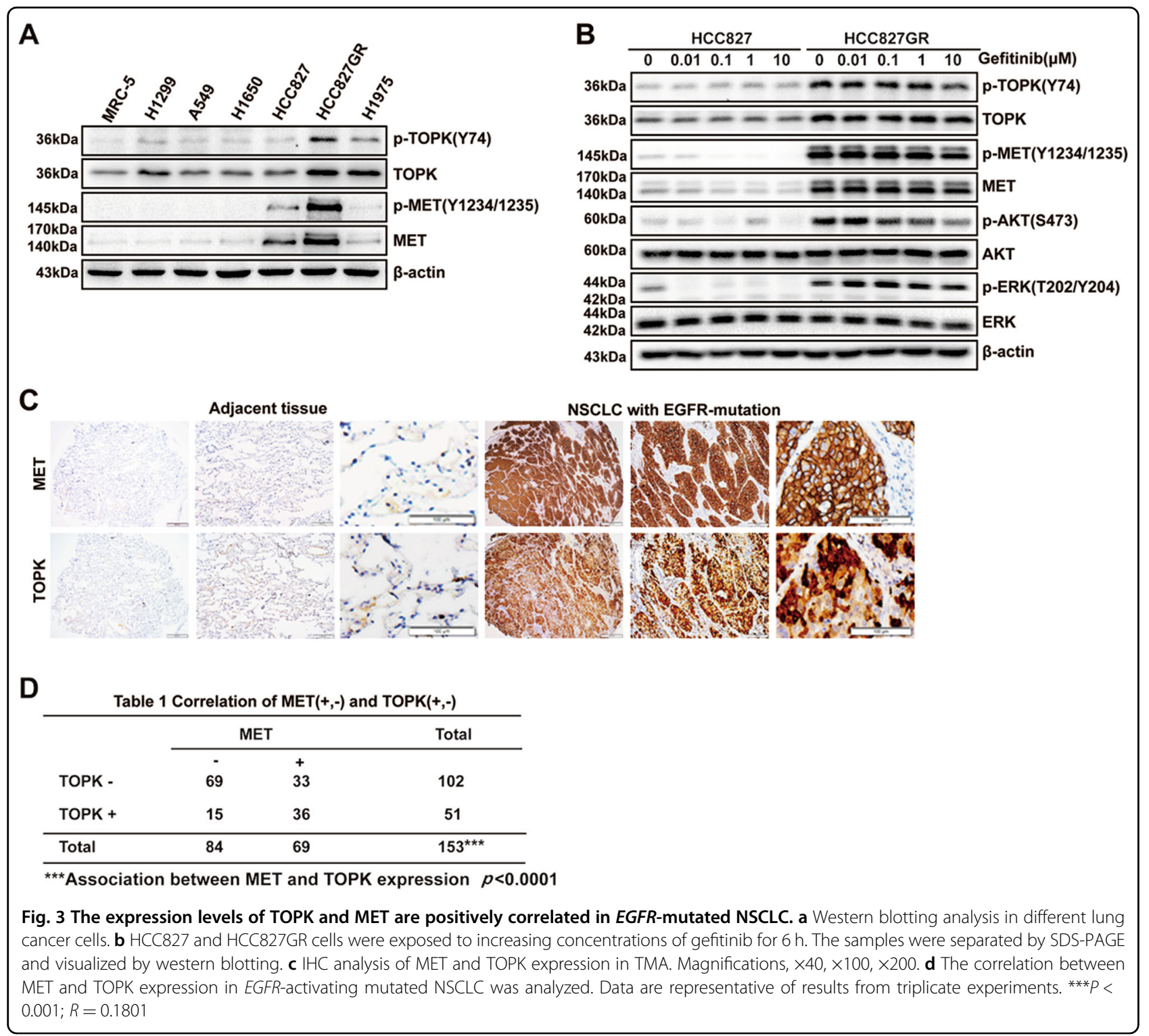

The COX2/MET/TOPK signaling axis inhibits cell apoptosis and promotes the anchorage-independent growth ability of HCC827 cells

From the above results, we found that COX2 regulated MET by AP-1 and MET phosphorylated TOPK at the Y74 site. Then, we further explored the effect of the COX2/MET/TOPK signaling pathway on gefitinib resistance. The cleavage of PARP (a marker of apoptosis) was increased in knockdown COX2 or TOPK HCC827GR cells after $1 \mu \mathrm{M}$ gefitinib treatment for $24 \mathrm{~h}$ (Fig. 5a). Flow cytometry revealed that knockdown of COX2 or TOPK promoted apoptosis of HCC827GR cells (Fig. 5b, and Fig. S1A and S1B). Meanwhile, decreased PARP cleavage was seen in HCC827 cells stably overexpressing COX2 or TOPK after treatment with gefitinib $(1 \mu \mathrm{M}, 24 \mathrm{~h})$ (Fig. $5 \mathrm{c}$ ). The expression of cleaved PARP was recovered in the group stably overexpressing TOPK-74F (Fig. 5c, right). In addition, flow cytometry showed that overexpression of COX2 or TOPK in HCC827 cells inhibited cell apoptosis and the apoptosis rate of HCC827 cells stably overexpressing TOPK-74F was increased compared with HCC827 cells stably overexpressing TOPK after treatment with gefitinib $(1 \mu \mathrm{M}, 24 \mathrm{~h})$ (Fig. $5 \mathrm{~d}$, and Fig. S1C and S1D). Based on these data, the anchorage-independent colony-formation ability of stable cell lines was then tested. The results showed that gefitinib strongly inhibited HCC827 (Mock) cell growth as expected at different concentrations $(0.5 \mu \mathrm{M}, 1 \mu \mathrm{M}$, or $2 \mu \mathrm{M})$. Compared with the control group, the colonies formed by $\mathrm{HCC} 827$ (COX2) cells were significantly greater in number and larger in size, and could not be entirely inhibited by gefitinib (Fig. 5e). Similarly, the colonies formed by 


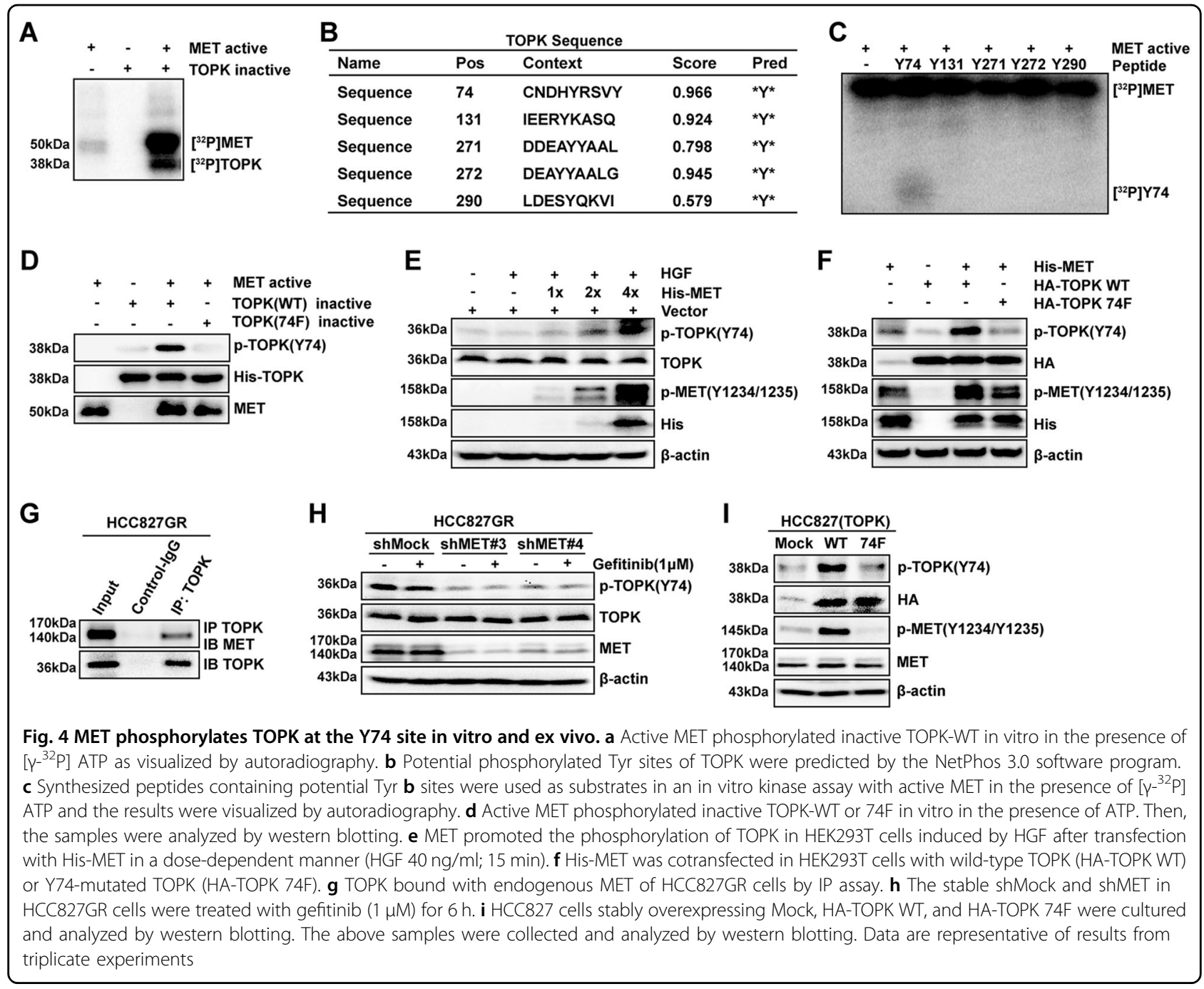

HCC827 (TOPK-WT) cells were also more numerous and larger in size than those formed by HCC827 (Mock) cells with or without gefitinib treatment. Moreover, fewer and smaller colonies were formed by the HCC827 (TOPK74F) cells (Fig. 5f). These results indicated that COX2 and TOPK could inhibit the apoptosis of HCC 827 cells and promote the anchorage-independent growth ability of HCC827 cells. This suggests that the inhibition of COX2 and TOPK may overcome resistance to gefitinib.

The triple combination of drugs strongly suppresses cell growth and colony formation accompanied by induction of apoptosis in vitro and in vivo

As the inhibition of COX2 or TOPK could induce apoptosis of HCC827GR cells, we combined celecoxib (the COX2 inhibitor), pantoprazole (the TOPK inhibitor), and gefitinib in the following experiments. First, we assessed any potential cytotoxicity of gefitinib, celecoxib, and pantoprazole alone in HCC827GR cells (Fig. S2A). Then, combined effects were measured by MTT assay and analyzed using the Chou-Talalay method (Fig. S2B and C). The CI data showed that various concentrations of gefitinib, celecoxib, and pantoprazole induced different effects. The low-dose combination of gefitinib $(1 \mu \mathrm{M})$, celecoxib $(20 \mu \mathrm{M})$, and pantoprazole $(100 \mu \mathrm{M})$ achieved a synergistic effect on proliferation inhibition in HCC827GR cells (Fig. S2C and Fig. 6a). As shown in Fig. 6b, this triple combination resulted in significantly higher inhibition of cell growth relative to any of the double combinations or single-drug treatments over $72 \mathrm{~h}$. Subsequently, the triple treatment led to a significant induction in apoptosis as measured by PARP cleavage (Fig. 6c). Moreover, as expected, the triple combination was also shown to significantly inhibit the colony-formation ability of HCC827GR cells by using a soft agar assay (Fig. 6d). These results showed that the triple combination of drugs could suppress cell growth and colony formation accompanied by induction of apoptosis in vitro. 


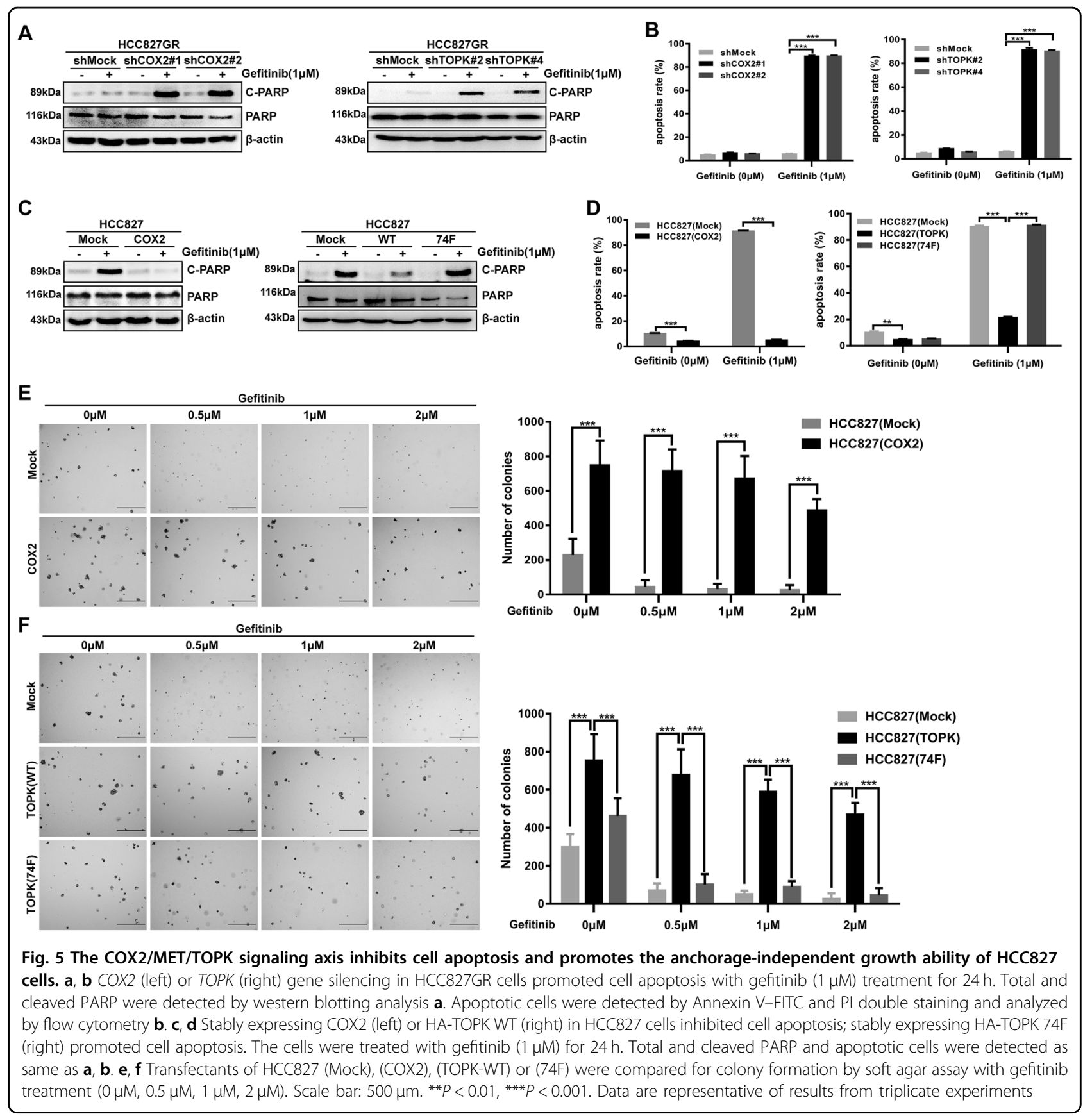

Based on the above results, we performed an animal study to test the effectiveness of the triple combination in preventing HCC827GR tumor growth. The results showed that the triple combination of gefitinib, celecoxib, and pantoprazole suppressed HCC827GR-mediated tumor growth (Fig. 6e, f). In addition, through retrospective analysis of 153 patients with EGFR-activating mutations, we identified that the PFS of gefitinib-resistant patients who were COX2, MET, and TOPK triple-positive was shorter than that of patients who were triple-negative (Fig. 6g). These results indicated that triple-positive patients with COX2, MET, and TOPK had a worse prognosis. Taken together, our study suggests that the triple combination of celecoxib, gefitinib, and pantoprazole can overcome gefitinib resistance.

\section{Discussion}

It was reported that some factors, including hypoxiainduced factors, cytokines, HGF-dependent autocrine or paracrine loops, or proangiogenic factors, contributed to the transcriptional upregulation of $\mathrm{MET}^{22,23}$. From our data, overexpression of COX2 regulating MET expression 


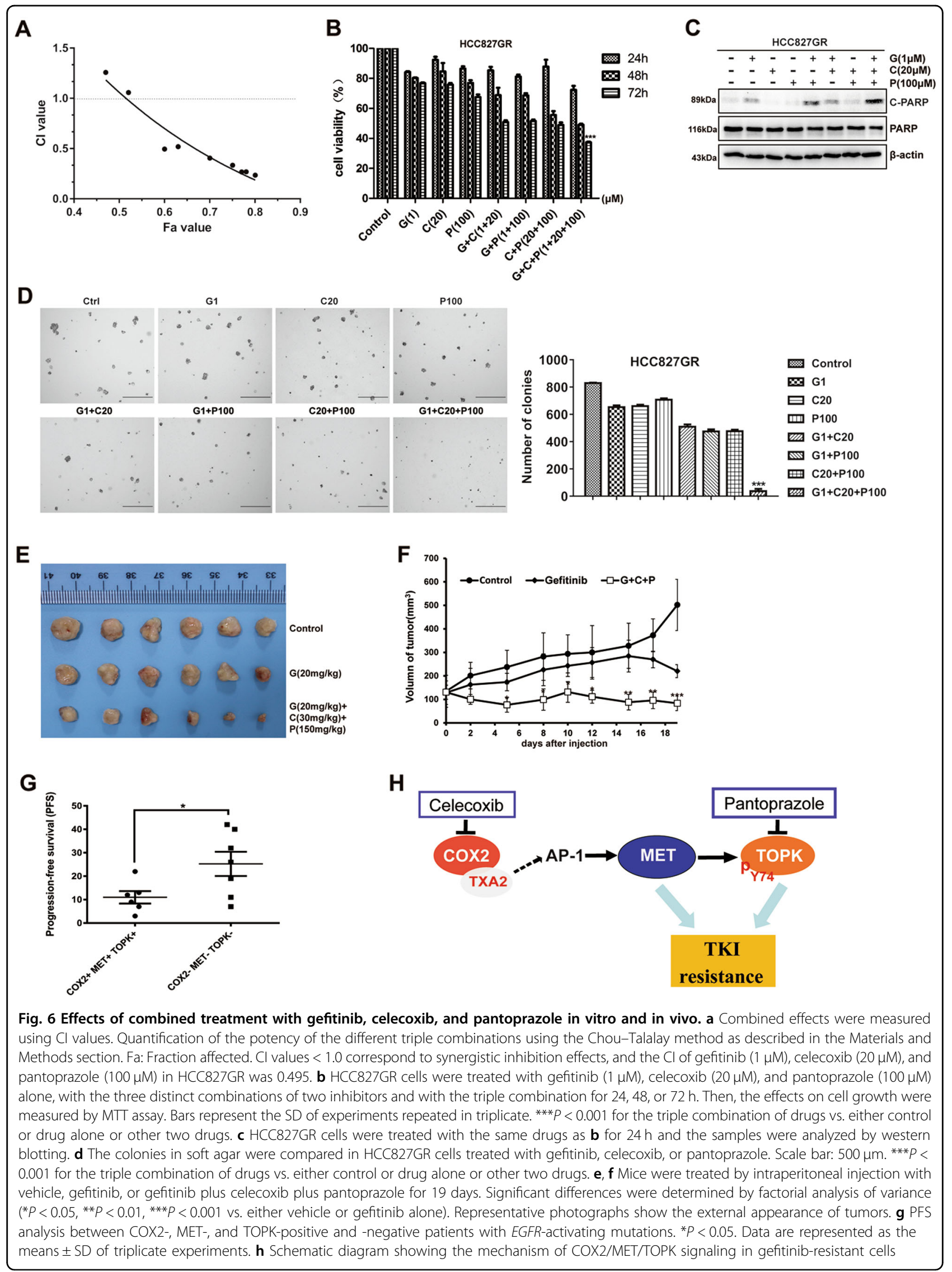


at the transcriptional level via AP-1 induced gefitinib resistance. In addition, COX2 has served as a potential therapeutic target for lung cancer and selective COX2 inhibitors such as celecoxib were developed for use in lung cancer treatment and prevention ${ }^{10}$. Compared with those regulators of MET, the expression of $\mathrm{COX} 2$ could be a biomarker for gefitinib-resistant NSCLC with MET overexpression and $\mathrm{COX} 2$ could also be a target for treating gefitinib resistance.

In addition, COX2 is involved in various aspects of cancer primarily through $\mathrm{PG}$ synthesis and $\mathrm{PGE}_{2}$ might be the predominant PG in cancer ${ }^{9}$. However, in our study, $\mathrm{TXA}_{2}$ was the most represented PG in gefitinib-resistant cells compared with the other four major bioactive PGs and the COX2-TXA 2 pathway-mediated MET transcription. It was reported that $\mathrm{TXA}_{2}$ or TXAS had been established as a tumor promoter in colon cancer or lung cancer $^{24}$. Li et al. ${ }^{25}$ established that CRC progression is accompanied by elevated TXA 2 levels and indicated that circulating $\mathrm{TXA}_{2}$ levels might have a potential prognostic or predictive value for the early detection of CRC. Hence, our findings suggested that $\mathrm{TXA}_{2}$ levels might have a potential predictive value in gefitinib-resistant NSCLC.

Drug resistance caused by MET overactivation is an urgent problem to be solved in the treatment of lung cancer. To date, some MET-targeted agents such as SU11274 and crizotinib have been developed and used in clinical experiments ${ }^{26}$. However, there have not been any useful drugs entering the market as first-line therapies due to negative or disappointing clinical trials ${ }^{27}$. One reason is that there are no appropriate clinical indicators for selecting patients with MET overactivation for treatment. MET protein expression was considered to have low predictive value, because the IHC assay lacked an established standard or consensus on optimized cutoff values and the total MET might not always reflect the activation of MET signaling ${ }^{28}$. In addition, only a few studies have investigated the expression of phosphorylated MET in lung cancer. Tretiakova et al. ${ }^{29}$ reported that the expression of two specific forms of p-MET (Y1003/Y1365) was associated with significantly worse overall survival. MET phosphorylation at Y1234/1235 and Y1349 could be detected in the NSCLC tumor samples, but there was no significant correlation between MET expression and pMET (Y1234/1235 or Y1349) expression ${ }^{30}$. None of them could be used to select patients for targeting therapies. In our study, we showed that MET could be regulated by COX2 and could phosphorylate and activate TOPK at the Y74 site. Concurrently, the new COX2/MET/TOPK signaling axis promoted gefitinib resistance. Moreover, the PFS of COX2/MET/TOPK triple-positive patients with gefitinib resistance was shorter than that of triple-negative patients. In addition, the triple-positive patients with
COX2, MET, and TOPK had a worse prognosis. Therefore, COX2 and TOPK expression can be associated with MET expression as predictive and prognostic markers for patients with gefitinib resistance.

The other reason that there is no useful treatment is that it is costly and time-consuming to explore new drugs for EGFR-TKI resistance. Our results strongly suggested that the combined inhibition of COX2, MET, and TOPK could overcome gefitinib resistance in HCC827GR cells, and that this strategy would be superior to targeting an individual pathway. Both celecoxib and pantoprazole are FDA-approved common drugs. Celecoxib, as a nonsteroidal anti-inflammatory drug, was reported to be used in the treatment of NSCLC due to the relationship between cancer and inflammation ${ }^{31}$. Moreover, pantoprazole was considered to increase the sensitivity of drugresistant cancer cells such as lymphomas and gastric adenocarcinomas $^{32}$. In our study, the triple combination approach with lower celecoxib, pantoprazole, and gefitinib concentrations effectively inhibited the tumorigenesis of gefitinib-resistant cells. Therefore, the new triple combination would provide a low-cost and practical strategy to help gefitinib-resistant patients.

In summary, our findings reveal a vital COX2/MET/ TOPK signaling axis inducing gefitinib resistance in NSCLC and propose a low-cost and practical strategy to overcome this problem. In this regard, it might be useful to verify the effect of the triple combination of celecoxib, pantoprazole, and gefitinib through multicenter clinical trials, and to detect the $\mathrm{TXA}_{2}$ level in plasma, pleural effusions, or tissues of NSCLC patients and evaluate its clinical value.

\footnotetext{
Acknowledgements

We are grateful to Mee-Hyun Lee, Shanshan Huang, and Chuntao Quan for their comments and technical support with this project. This work was supported by the National Natural Science Foundation of China (Numbers 81672936, 81672739, 81503105, and 81472602), the Independent innovation project of Huazhong University of Science and Technology (Numbers 2018KFYYXJJ084, 2016YXZD034, and 2015QN151), and Hubei Province Health and Family Planning Scientific Research Project (Numbers WJ2019M109 and WJ2017M050).
}

\begin{abstract}
Author details
${ }^{1}$ Department of Biochemistry and Molecular Biology, School of Basic Medicine, Huazhong University of Science and Technology, Wuhan, Hubei 430030, China. ${ }^{2}$ Cancer Research Institute, The Affiliated Hospital of Guilin Medical University, Guilin, Guangxi 541000, China. ${ }^{3}$ Department of Pathology, School of Basic Medicine, Huazhong University of Science and Technology, Wuhan 430030, China. ${ }^{4}$ Institute of Pathology, Tongji Hospital, Huazhong University of Science and Technology, Wuhan 430030, China. ${ }^{5}$ Department of General Surgery, The First Affiliated Hospital of Zhengzhou University, Zhengzhou 450052, China. ${ }^{6}$ Internal Medicine, CR\&WISCO General Hospital Affiliated to Wuhan University of Science and Technology, Wuhan 430080, China. ${ }^{7}$ Key Lab of Birth Defects and Reproductive Health of National Health and Family Planning Commission, Chongqing Population and Family Planning Science and Technology Research Institute, Chongqing 400020, China. ${ }^{8}$ Henan International Joint Laboratory for Nuclear Protein Regulation, Henan University College of Medicine, Kaifeng, Henan 475004, China. ${ }^{9}$ College of Food Science and Nutritional Engineering,
\end{abstract}


China Agricultural University, Beijing 100083, China. ${ }^{10}$ Department of Pathology, National Cancer Center/National Clinical Research Center for Cancer/Cancer Hospital, Chinese Academy of Medical Sciences and Peking Union Medical College, Beijing 100021, China. ${ }^{11}$ Department of Pathology, Union Hospital, Huazhong University of Science and Technology, Wuhan, Hubei 430030, China. ${ }^{12}$ Department of Oncology, Tongji Hospital, Huazhong University of Science and Technology, Wuhan, Hubei, China

\section{Conflict of interest}

The authors declare that they have no conflict of interest.

\section{Publisher's note}

Springer Nature remains neutral with regard to jurisdictional claims in published maps and institutional affiliations.

Supplementary Information accompanies this paper at (https://doi.org/ 10.1038/s41419-019-2020-4).

Received: 14 May 2019 Revised: 20 September 2019 Accepted: 26 September 2019

Published online: 14 October 2019

\section{References}

1. Siegel, R. L., Miller, K. D. \& Jemal, A. Cancer statistics, 2018. Cancer J. Clin. 68 7-30 (2018)

2. Shi, Y. et al. A prospective, molecular epidemiology study of EGFR mutations in Asian patients with advanced non-small-cell lung cancer of adenocarcinoma histology (PIONEER). J. Thorac. Oncol. 9, 154-162 (2014).

3. Wu, S. G. \& Shih, J. Y. Management of acquired resistance to EGFR TKItargeted therapy in advanced non-small cell lung cancer. Mol. Cancer 17, 38 (2018).

4. Gelsomino, F. et al. Targeting the MET gene for the treatment of non-smallcell lung cancer. Crit. Rev. Oncol. Hematol. 89, 284-299 (2014).

5. Ko, B., He, T., Gadgeel, S. \& Halmos, B. MET/HGF pathway activation as a paradigm of resistance to targeted therapies. Ann. Transl. Med. 5, 4 (2017).

6. Hui, A. Y. et al. Src and FAK mediate cell-matrix adhesion-dependent activation of Met during transformation of breast epithelial cells. J. Cell. Biochem. 107, 1168-1181 (2009)

7. Dulak, A. M., Gubish, C. T., Stabile, L. P., Henry, C. \& Siegfried, J. M. HGFindependent potentiation of EGFR action by c-Met. Oncogene 30, 3625-3635 (2011).

8. Graveel, C. R., Tolbert, D. \& Vande Woude, G. F. MET: a critical player in tumorigenesis and therapeutic target. Cold Spring Harb. Perspect. Biol. 5, pii: a009209 (2013).

9. Misra, S. \& Sharma, K. COX-2 signaling and cancer: new players in old arena. Curr. Drug Targets 15, 347-359 (2014).

10. Liu, R., Xu, K. P. \& Tan, G. S. Cyclooxygenase-2 inhibitors in lung cancer treatment: bench to bed. Eur. J. Pharmacol. 769, 127-133 (2015).

11. $\mathrm{Li}, \mathrm{H}$. et al. Aspirin prevents colorectal cancer by normalizing EGFR expression. EBioMedicine 2, 447-455 (2015).
12. Seol, D. W., Chen, Q. Y. \& Zarnegar, R. Transcriptional activation of the hepatocyte growth factor receptor (c-met) gene by its ligand (hepatocyte growth factor) is mediated through AP-1. Oncogene 19, 1132-1137 (2000).

13. Lei, B. et al. PBK/TOPK expression in non-small-cell lung cancer: its correlation and prognostic significance with Ki67 and p53 expression. Histopathology $\mathbf{6 3}$, 696-703 (2013).

14. Li, Y. et al. TOPK promotes lung cancer resistance to EGFR tyrosine kinase inhibitors by phosphorylating and activating c-Jun. Oncotarget 7, 6748-6764 (2016).

15. Xiao, J. J. et al. Phosphorylation of TOPK at Y74, Y272 by Src increases the stability of TOPK and promotes tumorigenesis of colon cancer. Oncotarget 7, 24483-24494 (2016).

16. McAdam, B. F. et al. Systemic biosynthesis of prostacyclin by cyclooxygenase (COX)-2: The human pharmacology of a selective inhibitor of COX-2. Proc. Natl Acad. Sci. USA 96, 272-277 (1999).

17. Zeng, X. Y. et al. Pantoprazole, an FDA-approved proton-pump inhibitor, suppresses colorectal cancer growth by targeting T-cell-originated protein kinase. Oncotarget 7, 22460-22473 (2016).

18. Engelman, J. A. et al. MET amplification leads to gefitinib resistance in lung cancer by activating ERBB3 signaling. Science 316, 1039-1043 (2007).

19. Chou, T. C. Drug combination studies and their synergy quantification using the Chou-Talalay method. Cancer Res. 70, 440-446 (2010).

20. Menter, D. G., Schilsky, R. L. \& DuBois, R. N. Cyclooxygenase-2 and cancer treatment: understanding the risk should be worth the reward. Clin. Cancer Res. 16, 1384-1390 (2010).

21. Bode, M. \& Mackman, N. Regulation of tissue factor gene expression in monocytes and endothelial cells: thromboxane A2 as a new player. Vasc. Pharmacol. 62, 57-62 (2014).

22. Organ, S. L. \& Tsao, M. S. An overview of the c-MET signaling pathway. Therapeutic Adv. Med. Oncol. 3, S7-S19 (2011).

23. Zhang, J. \& Babic, A. Regulation of the MET oncogene: molecular mechanisms. Carcinogenesis 37, 345-355 (2016).

24. Huang, Q. C. \& Huang, R. Y. The cyclooxygenase-2/thromboxane A2 pathway: a bridge from rheumatoid arthritis to lung cancer? Cancer Lett. 354, 28-32 (2014).

25. Li, H. T. et al. Circulating prostaglandin biosynthesis in colorectal cancer and potential clinical significance. EBioMedicine 2, 165-171 (2015).

26. Feng, Y. \& Ma, P. C. MET targeted therapy for lung cancer: clinical development and future directions. Lung Cancer 3, 53-67 (2012).

27. Drilon, A., Cappuzzo, F., Ou, S. I. \& Camidge, D. R. Targeting MET in lung cancer: will expectations finally be MET? J. Thorac. Oncol. 12, 15-26 (2017).

28. Salgia, R. MET in lung cancer: biomarker selection based on scientific rationale. Mol. Cancer Ther. 16, 555-565 (2017).

29. Tretiakova, M. et al. MET and phosphorylated MET as potential biomarkers in lung cancer. J. Environ. Pathol. Tox 30, 341-354 (2011).

30. Watermann, I. et al. Improved diagnostics targeting c-MET in non-small cell lung cancer: expression, amplification and activation? Diagnostic Pathol. 10, 130 (2015).

31. Gulyas, M. et al. COX-2 expression and effects of celecoxib in addition to standard chemotherapy in advanced non-small cell lung cancer. Acta Oncol. 57, 244-250 (2018).

32. De Milito, A. et al. Proton pump inhibitors induce apoptosis of human B-cel tumors through a caspase-independent mechanism involving reactive oxygen species. Cancer Res. 67, 5408-5417 (2007). 